Supplement of

\title{
Organic aerosol source apportionment in London 2013 with ME-2: exploring the solution space with annual and seasonal analysis
}

Ernesto Reyes-Villegas et al.

Correspondence to: James D. Allan (james.allan@manchester.ac.uk)

The copyright of individual parts of the supplement might differ from the CC-BY 3.0 licence. 


\section{S1. PMF solutions to determine the number of sources.}

PMF runs (Fig S1) with different number of factors (sources) were performed to determine the number of OA sources. The six-factor solution (figure S1.c) shows two split factors (dark blue and green) which correspond to the same source, LVOOA. The five-factor solution (Figure S1.b) was able to separate two secondary organic aerosol sources in SVOOA and LVOOA showing to be the more acceptable number of sources.

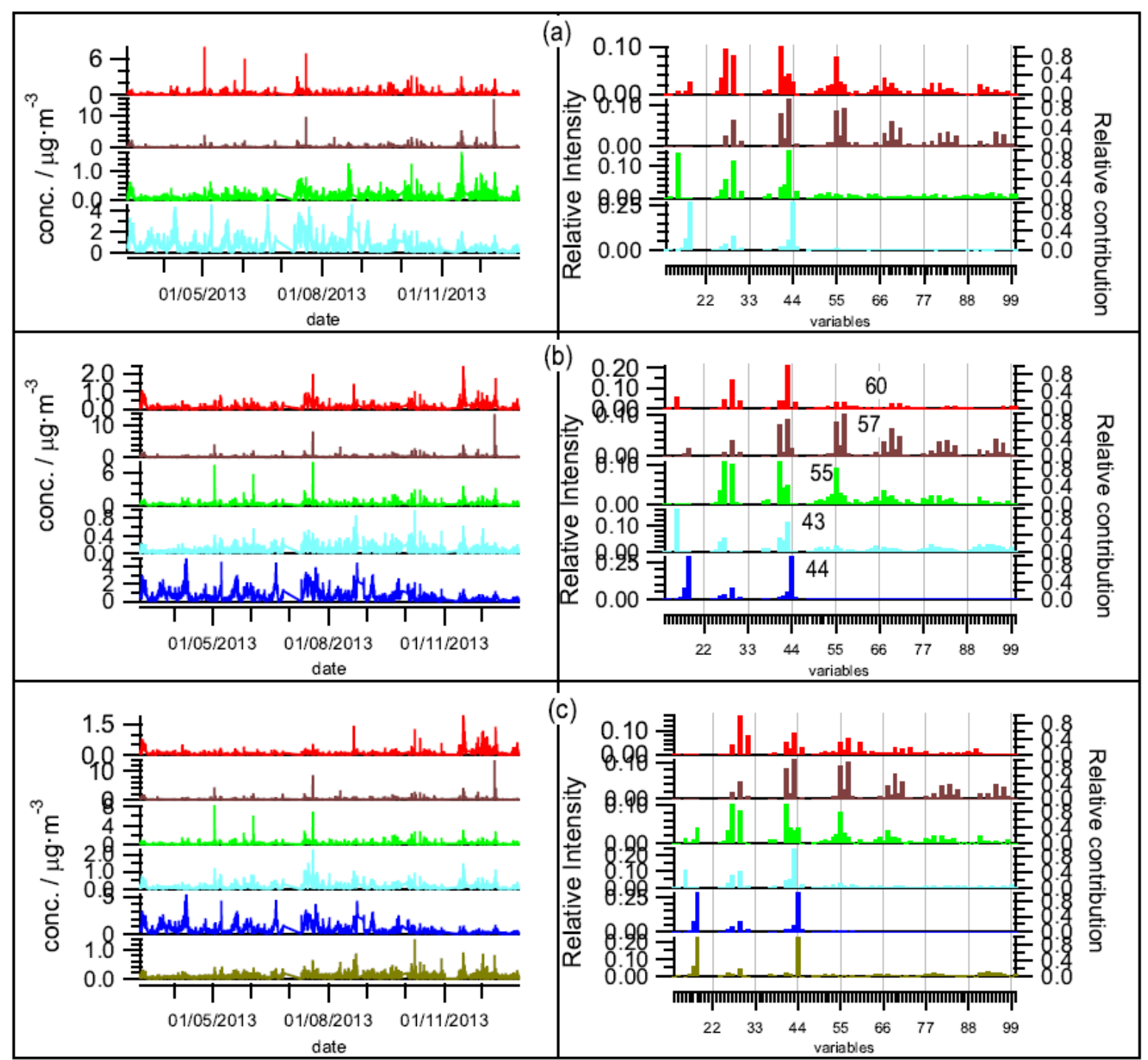

Figure S1: PMF solutions: four-factor solution (a), five-factor solution (b) and six-factor solution (c) to determine the number of $\mathrm{OA}$ sources. 


\section{S2. Seed and mass spectral analysis.}

In order to deal with rotational ambiguity, ME-2 runs may be initialised from different random values, also called seeds. Figure S2 shows the analysis performed to the three different seeds from the best solution chosen for March-December (aB3_H2_C3) to determine stability on the solutions. This stability proves that solutions may be repeatable with the three solutions presenting the same five factors with similar Q/Qexp (S2.a), mass spectrum (S2.b) and time series (S2.C).

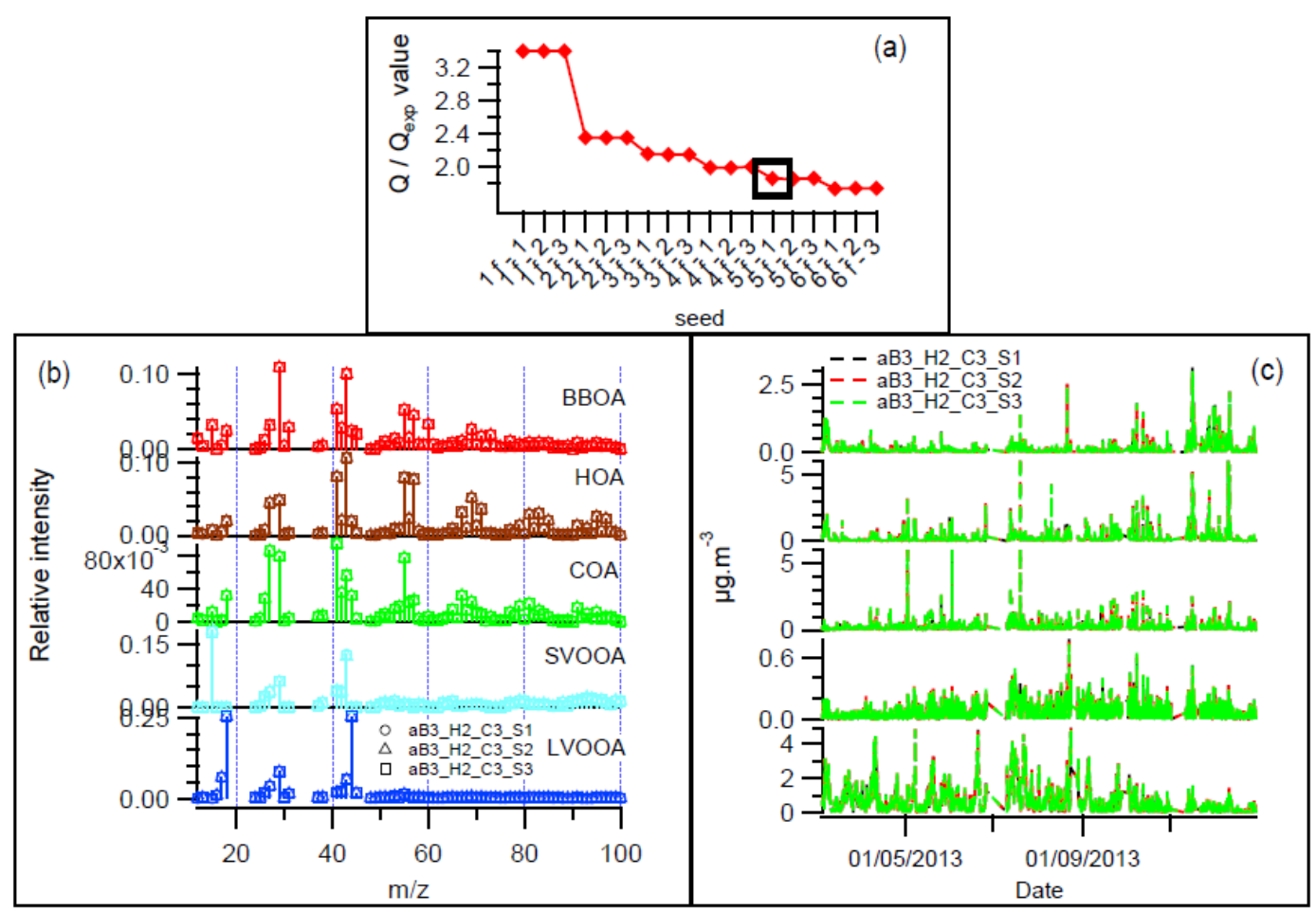

Figure S2: Seed analysis (a). Mass spectra (b) and time series (c).

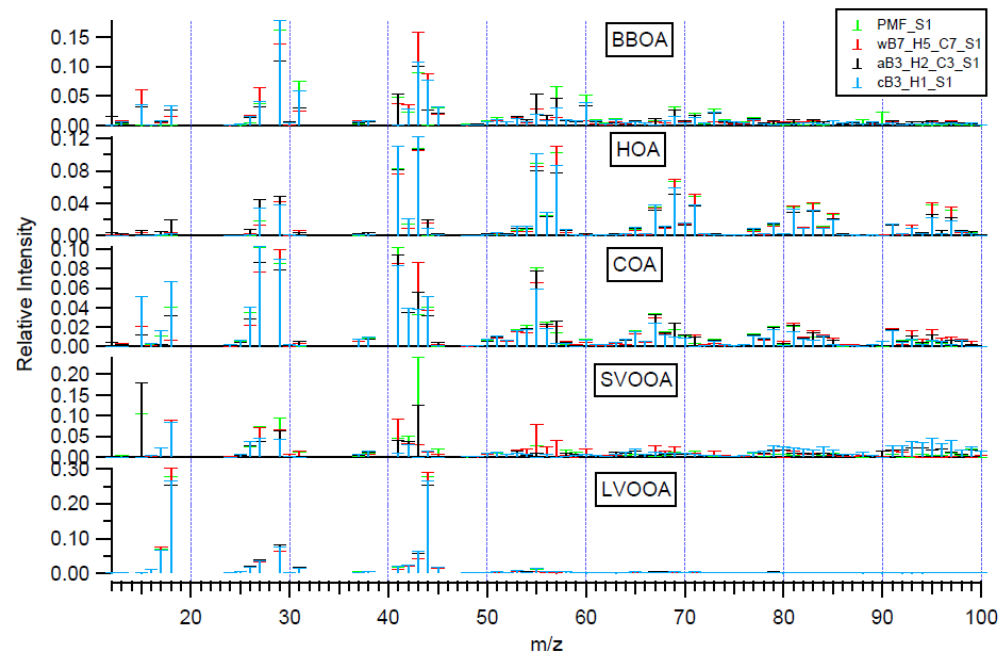

Figure S3: Mass spectra comparison for undesirable solutions for March-December analysis. Example of mass spectra of solutions with mixed factors for unconstrained and constrained solutions. 


\section{S3. Analysis to determine the best solution for the different periods of time.}

PMF runs were performed, for the March-December period, from fpeak - 1 to 1 with steps of 0.1 . Figure 4 shows the comparison of the runs that converged (some of the fpeaks did not converge) in order to determine the PMF solution that better identified the OA sources to be compared to the ME-2 solutions. Run number 4 is chosen to be the best solution, according to the statistical tests applied, with low diurnal residual and positive $\mathrm{COA}$ for $\mathrm{CO}$ and $\mathrm{BC}$ trilinear regressions.

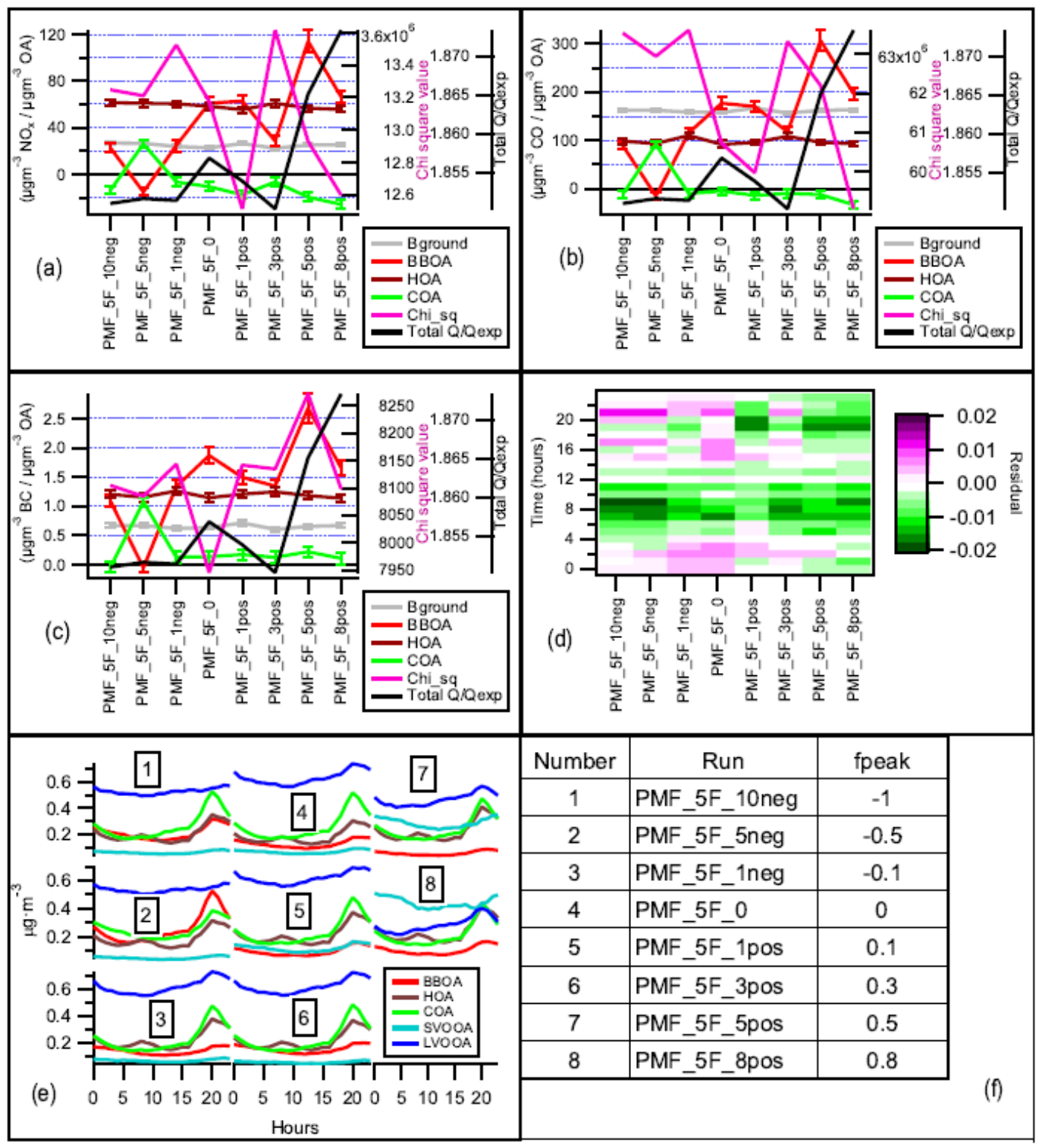

Figure S4 : NOx, CO and BC trilinear regression (a, b, c), diurnal residual (d), diurnal concentrations (e) and solution list for March-Dec PMF analysis (f). 
Figure S5 shows the analysis carried out to determine the best solution for the MarchDecember period. As mentioned in the main text of this paper, " $c$ " and " $w$ " target profiles (TP) show the less desirable results, "C" TP show a high positive residual (Figure 2.a) and "w" TP show a high chi-square and COA slope. (Figures C1.a and S4.b). From the "a" TP, aB3_H2_C3_S1 solution is chosen to present the best results from this analysis due to COA slope close to zero for NOx (Figure 2.b) and CO (Figure S5.a) trilinear regression and low diurnal residual (Figure 2.a).

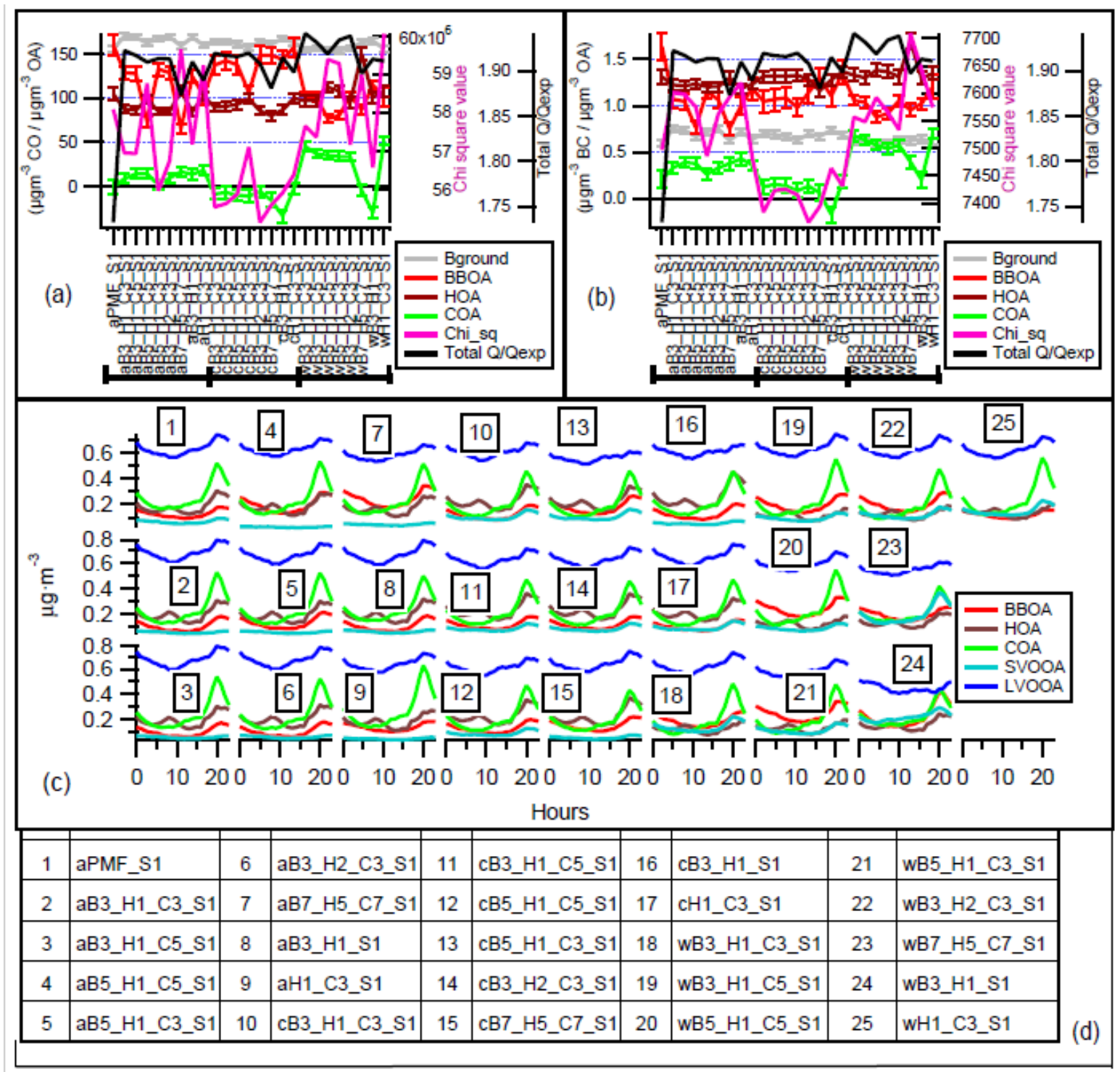

Figure S5: CO and BC trilinear regression (a, b), diurnal concentrations (c) and solution list for March-Dec analysis (d). 
Figure S6 shows the PMF analysis for the spring period. All solutions show similar diurnal concentrations with negative COA slope fo the three trilinear regressions. Solutions 2 and 3 have the lower Q/Qexp, Solution 3 was chosen to be compared with ME-2 solutions.

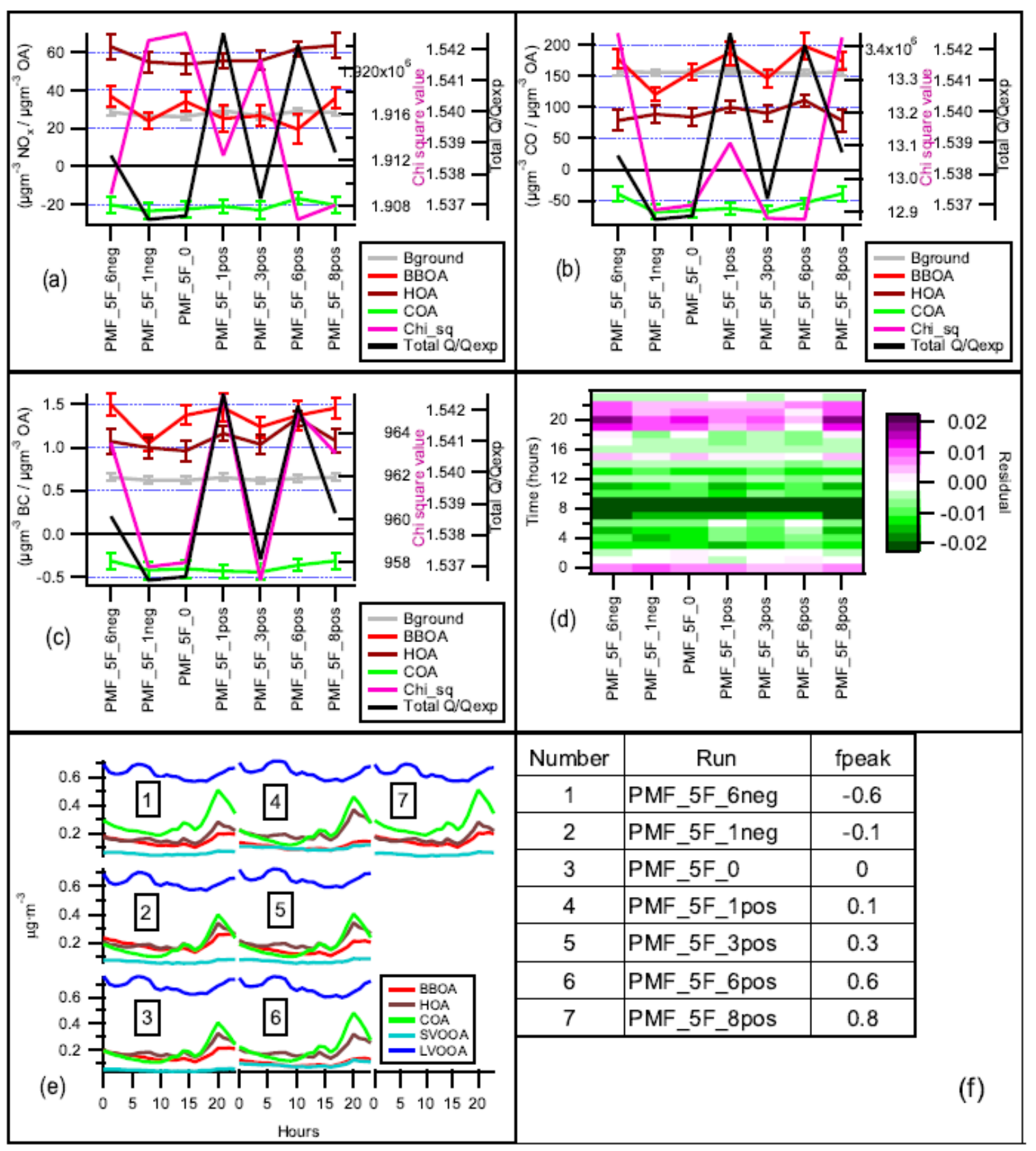

Figure S6: NOx, CO and BC trilinear regression (a, b, c), diurnal residual (d), diurnal concentrations (e) and solution list for spring PMF analysis (f). 
Figure S7 shows the analysis performed to determine the best solution for spring period. Solutions with "a" and "c" TP show the less desirable results with negative slopes for COA and high chi-square in the trilinear regression (Figures S5.a, S5.b and S5.c), "c" TP also show high diurnal residuals. The solution wB3_H1_C3_S1 is chosen to present the best results from this analysis with low chi-square and diurnal residuals.

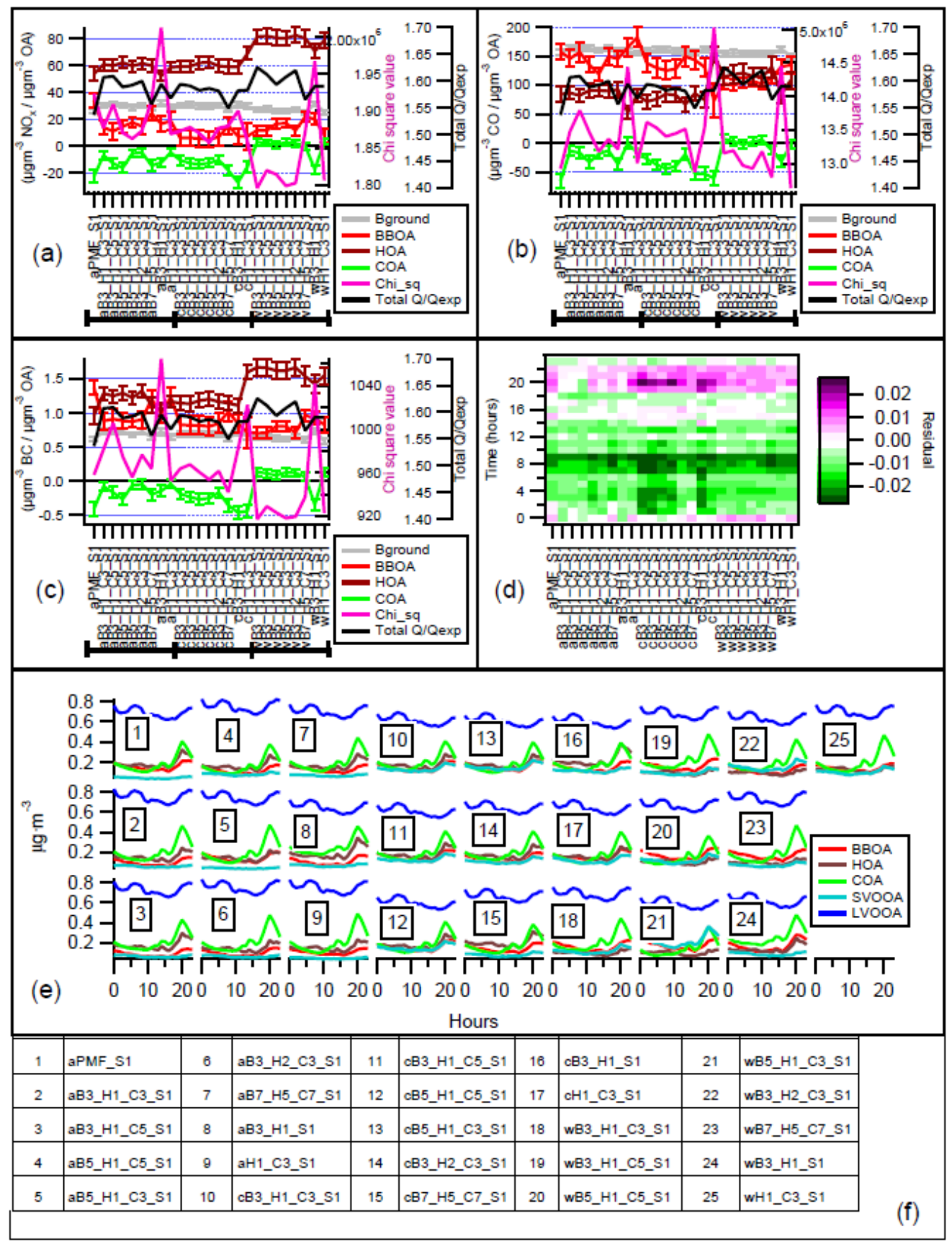

Figure S7: NOx, CO and BC trilinear regression (a,b,c), diurnal residual (d),diurnal concentrations (e) all the solutions for spring analysis (f). 
Figure S8 shows the PMF analysis for the summer period. Solution 4 has a high Q/Qexp but as it shows a COA slope close to zero in the three trilinear analyses and a low diurnal residual compared to the other PMF solutions, it has been chosen to be compared with ME2 solutions.

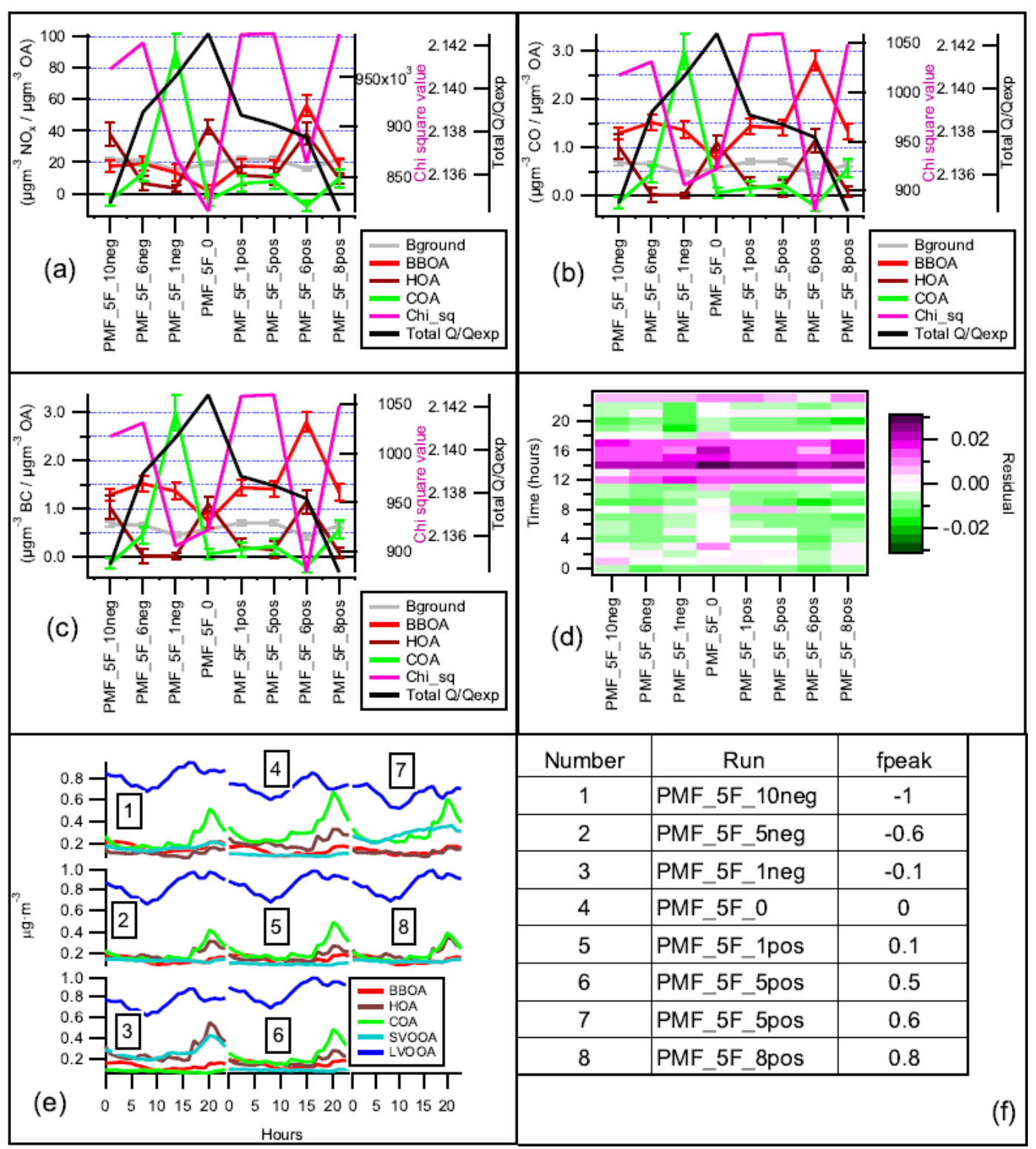

Figure S8: NOx, CO and BC trilinear regression (a, b, c), diurnal residual (d), diurnal concentrations (e) and solution list for summer PMF analysis (f). 
Figure S9 shows the analysis performed to determine the best solution for summer period. Solutions with "c" and "s" TP show the less desirable results. "s" TP show low chi-square values, however, they present high negative residuals in the morning and at night. "C" TP show a high positive residual around 15:00-18:00 hrs. The solution aB5_H1_C3_S1 is chosen to present the best results from this analysis due to the low diurnal residual, COA slope close to zero and the low BBOA slope in the NOx, BC and COA trilinear regressions (Figures S9.a, S9.b and S9.c).

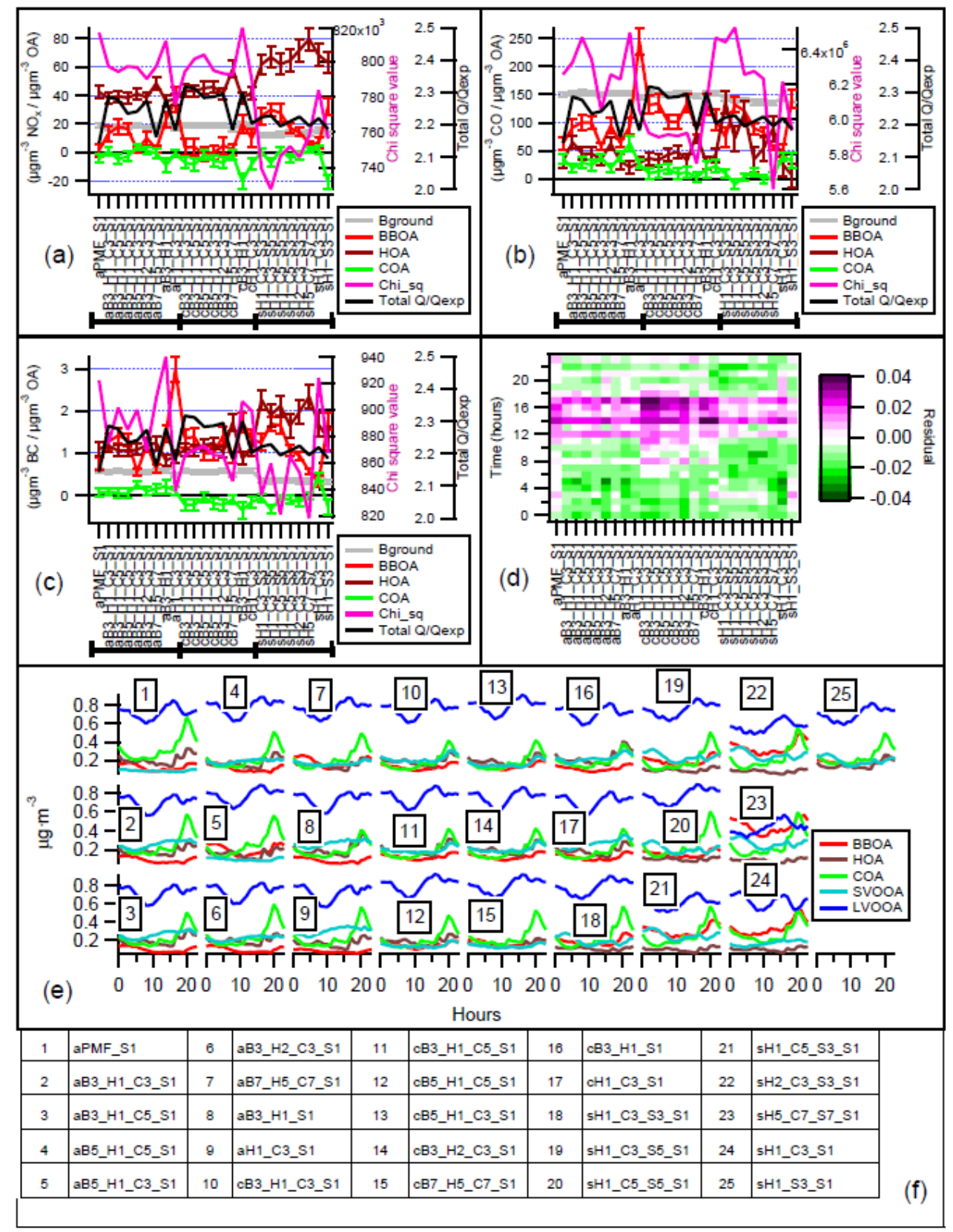

Figure S9: NOx, CO and BC trilinear regression (a,b,c), diurnal residual (d),diurnal concentrations (e) all the solutions for summer analysis (f). 
Figure S10 shows the PMF analysis for the autumn period. Solution 4 has been the chosen solution to be compared with ME-2 solutions because of its low Q/Qexp and a COA slope close to zero for the NOx trilinear regression and a lower diurnal residual compared to the other PMF solutions.

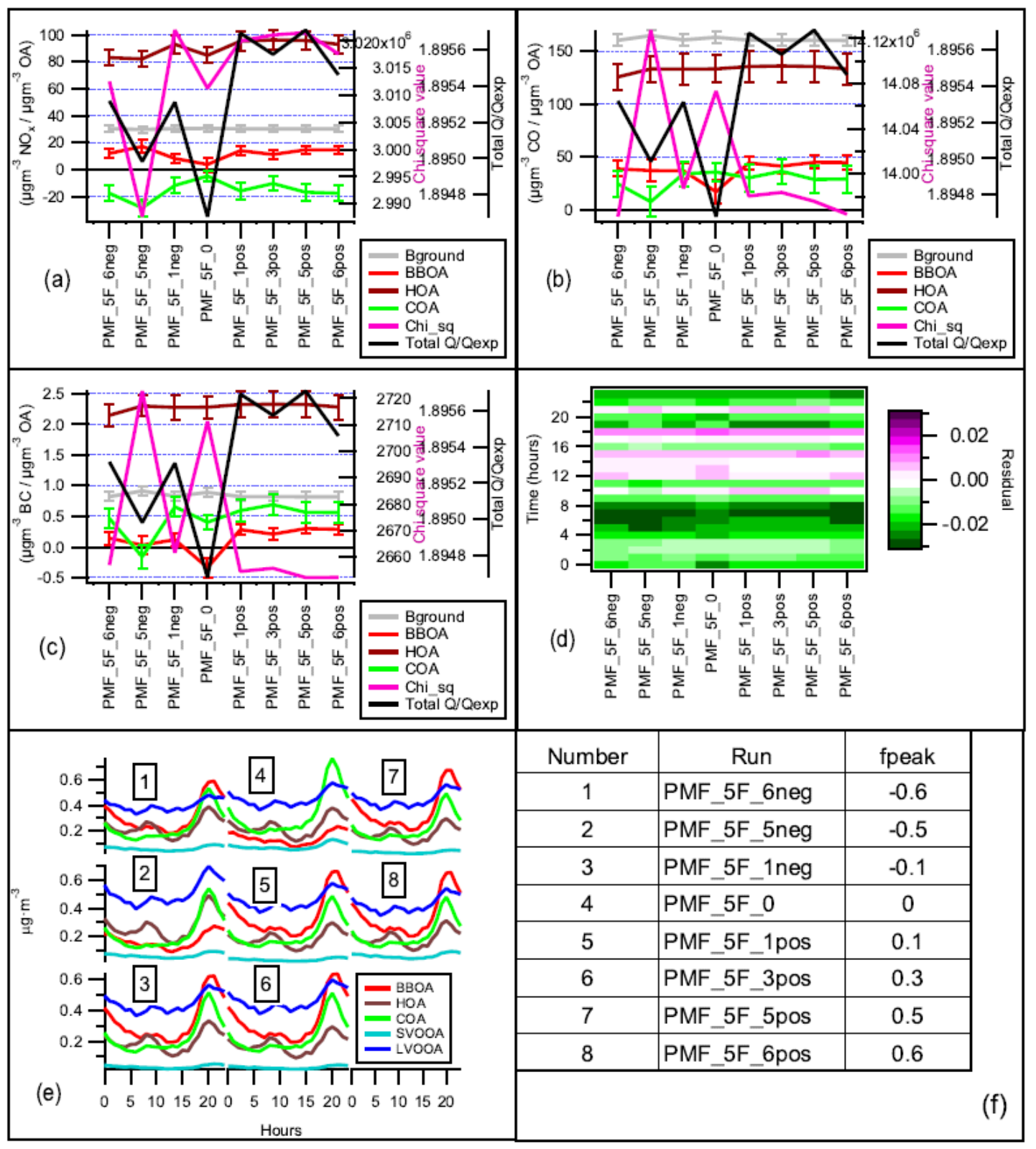

Figure S10: NOx, CO and BC trilinear regression (a, b, c), diurnal residual (d), diurnal concentrations (e) and solution list for autumn PMF analysis (f). 
Figure S11 shows the analysis performed to determine the best solution for autumn period. Solutions with "a" TP show the less favourable Chi square results in the three trilinear regression figures (Figures S11.a, S11.b and S11.C). WB3_H1_S1 solution is chosen to present the best results from this analysis with low chi-squares and COA slope close to zero in the trilinear regression with NOx (Figures S11.a).

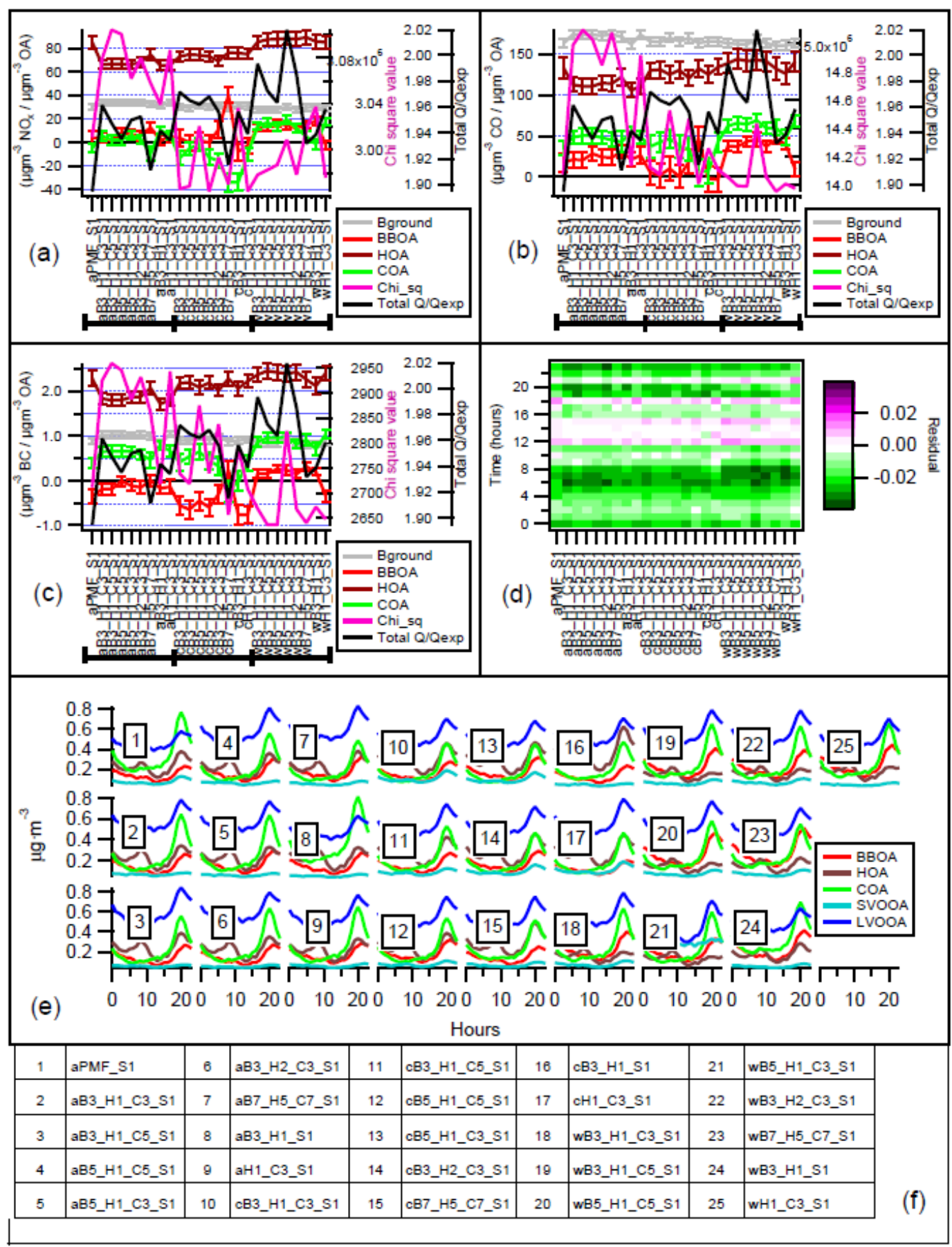

Figure S11: NOx, CO and BC trilinear regression (a,b,c), diurnal residual (d),diurnal concentrations (e) all the solutions for autumn analysis (f). 


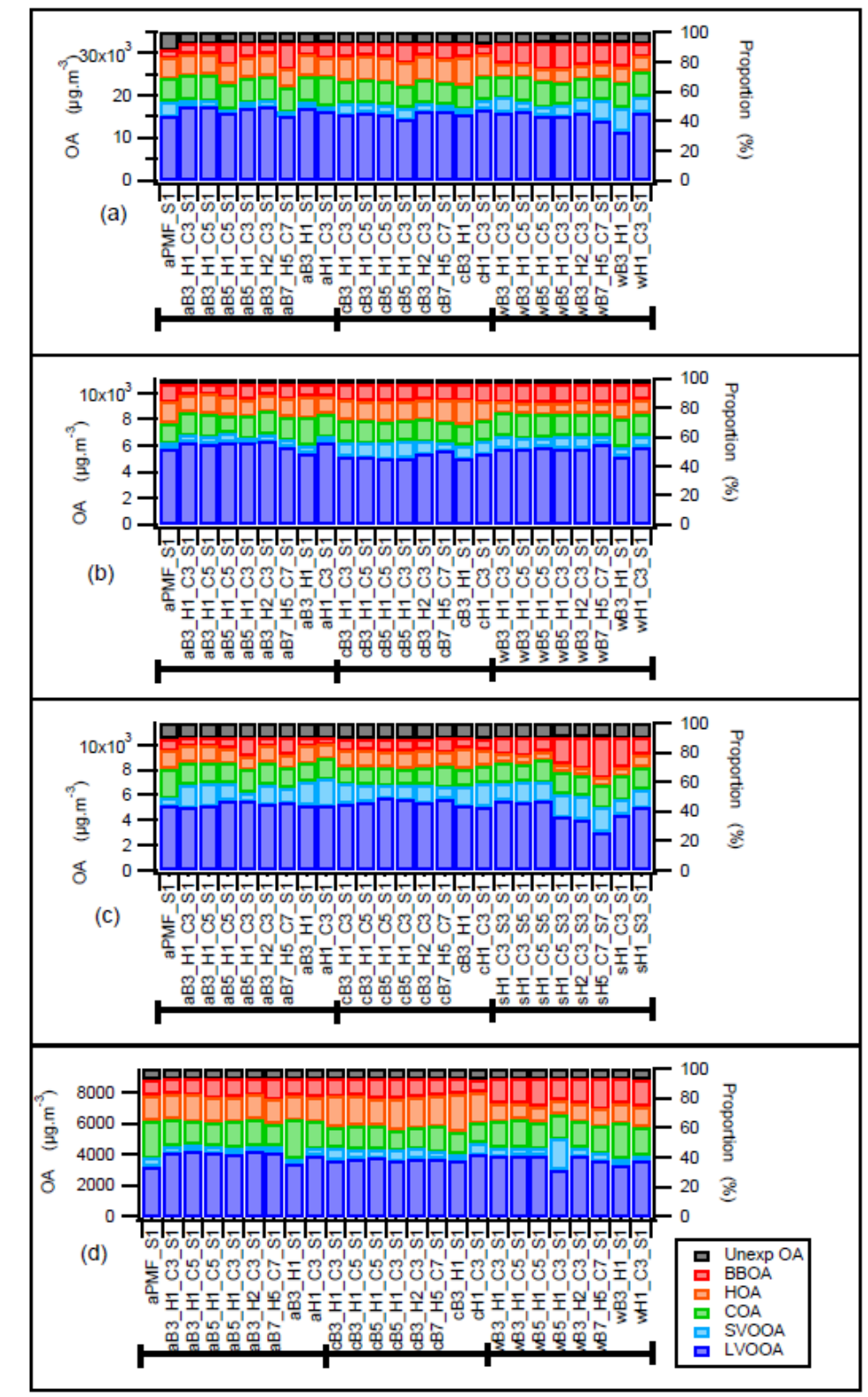

Figure S12: OA concentrations and proportions of the different OA sources to the total OA. March-Dec (a), spring (b), Summer (c) and autumn (d). 
S13. OA and meteorology time series to analyse $\mathrm{PM}_{2.5}$ concentrations.

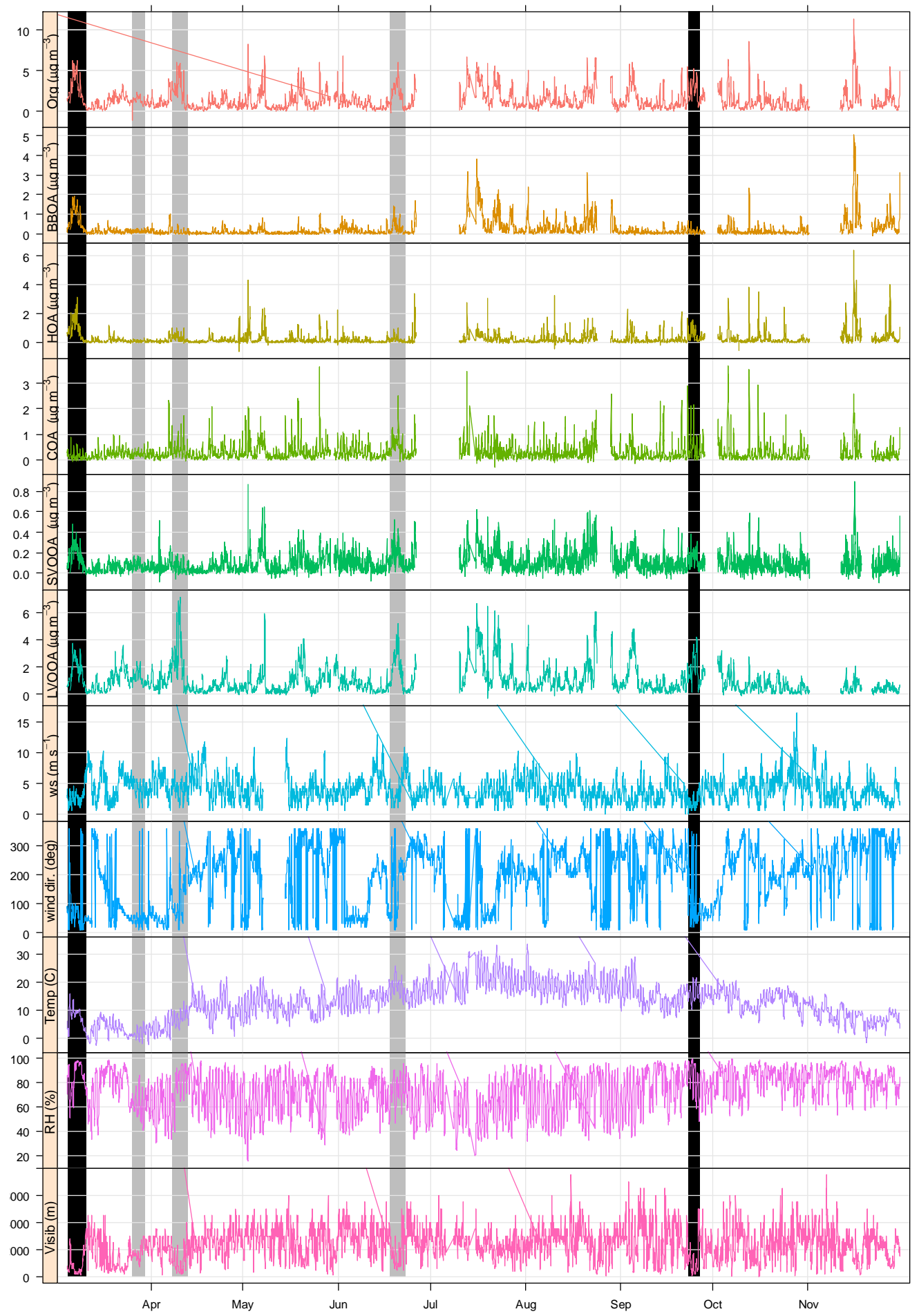

Figure S13: OA and meteorology showing moderate (grey) and high (black) $\mathbf{P M}_{2.5}$ concentrations. 